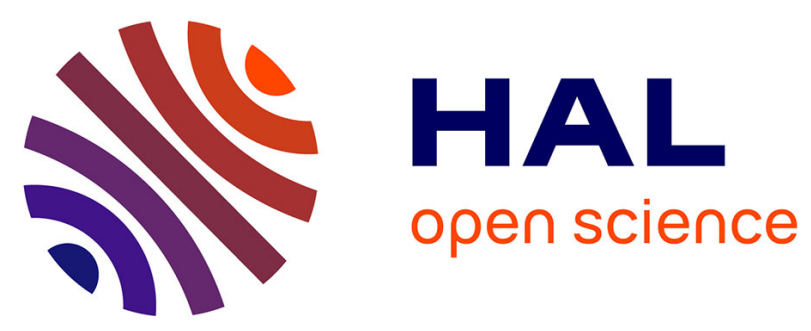

\title{
Bayesian analysis of individual and systematic multiplicative errors for estimating ages with stratigraphic constraints in optically stimulated luminescence dating
}

Benoit Combès, Anne Philippe

\section{To cite this version:}

Benoit Combès, Anne Philippe. Bayesian analysis of individual and systematic multiplicative errors for estimating ages with stratigraphic constraints in optically stimulated luminescence dating. 2016. hal-01346868v2

\author{
HAL Id: hal-01346868 \\ https://hal.science/hal-01346868v2
}

Preprint submitted on 30 Nov 2016

HAL is a multi-disciplinary open access archive for the deposit and dissemination of scientific research documents, whether they are published or not. The documents may come from teaching and research institutions in France or abroad, or from public or private research centers.
L'archive ouverte pluridisciplinaire HAL, est destinée au dépôt et à la diffusion de documents scientifiques de niveau recherche, publiés ou non, émanant des établissements d'enseignement et de recherche français ou étrangers, des laboratoires publics ou privés. 


\title{
Bayesian analysis of individual and systematic multiplicative errors for estimating ages with stratigraphic constraints in optically stimulated luminescence dating
}

\author{
Benoit Combès ${ }^{\mathrm{a}}$, Anne Philippe $\mathrm{b}^{\mathrm{b}, *}$ \\ ${ }^{a}$ IRAMAT-CRP2A, Maison de l'archéologie, Université de Bordeaux Montaigne, France. \\ ${ }^{b}$ Laboratoire de Mathématiques Jean Leray et ANJA INRIA - Rennes, Université de Nantes, France.
}

\begin{abstract}
Many dating techniques include significant error terms which are not independent between samples to date. This is typically the case in Optically Stimulated Luminescence (OSL) dating where the conversion from characteristic equivalent doses to the corresponding ages using the annual dosimetry data includes error terms that are common to all produced datings. Dealing with these errors is essential to estimate ages from a set of datings whose chronological ordering is known. In this work, we propose and we study a Bayesian model to address this problem. For this purpose, we first consider a multivariate model with multiplicative Gaussian errors in a Bayesian framework. This model relates a set of characteristic equivalent doses to the corresponding ages while taking into account for the systematic and non-systematic errors associated to the dosimetry. It thus offers the opportunity to deal properly with stratigraphic constraints within OSL datings, but also with other datings possessing errors which are independent from systematic errors of OSL (e.g. radiocarbon). Then, we use this model to extend an existing Bayesian model for the assessment of characteristic equivalent doses from Single Aliquot and Regenerative (SAR) dose measurements. The overall Bayesian model leads to the joint estimation of all the variables (which include all the dose-response functions and characteristic equivalent doses) of a sequence of, possibly heterogeneous, datings. We also consider a more generic solution consisting in using directly the age model from a set of characteristic equivalent dose
\end{abstract}

\footnotetext{
${ }^{*}$ Corresponding author. Anne.Philippe@univ-nantes.fr
} 
estimates and their associated standard errors. We finally give an example of application on a set of five OSL datings with stratigraphic constraints and observe a good adequacy between the two approaches.

Keywords: optically stimulated luminescence, chronometric dating, Bayesian analysis

\section{Introduction}

Ordering (or stratigraphic) constraints between samples to date constitute an essential element to build accurate and coherent chronologies. Such a prior information, typically modeled in a Bayesian setting [5], consists of a basic element of several dedicated softwares e.g. OxCal [3], BCal [4] or Chronomodel [12, 20]. The associated models are typically designed to deal with datings associated to additive and independent errors, as it is the case for e.g. radiocarbon or archaeomagnetic datings. However, paleodosimetric datings do not fit such a frame and, to the best of our knowledge, there is still a lack of generic methods to incorporate them in analyses. Paleodosimetric dating methods [18] rely on the assessment of a characteristic equivalent dose $D$ expressing the total absorbed radiation dose since last resetting event (e.g. last exposure to sunlight or heat) of the studied samples. Then, this characteristic equivalent dose can be converted into an age $A$ using the annual dose-rate $\dot{d}$ associated to the environment surrounding the studied sample. Given a characteristic equivalent dose estimate $\hat{D}$ and its standard error $\sigma_{D}$, the common procedure to perform this conversion consists in [17]:

1. summarizing the distribution of the annual dose-rate $\dot{d}$ with a mean $\mu_{\dot{d}}$ and standard error $\sigma_{\dot{d}}$ using combination rules,

2. approximating the point estimate and standard error for the associated age defined by the ratio $A=\frac{D}{\dot{d}}$ using the following quadrature formula:

$$
\hat{A}=\frac{\hat{D}}{\mu_{\dot{d}}} \text { and } \sigma_{A}=\hat{A} \sqrt{\left(\frac{\sigma_{D}}{\hat{D}}\right)^{2}+\left(\frac{\sigma_{\dot{d}}}{\mu_{\dot{d}}}\right)^{2}} \text {. }
$$

This solution has two main limitations: 
A. it provides only a coarse characterization of the statistics of $A$,

B. it does not permit to include additional information as ordering constraints between ages of a given stratigraphic sequence to improve the estimations. Moreover in practice, the dose-rate standard error $\sigma_{\dot{d}_{i}}$ for each dating $i$ includes components that are common to all the datings (i.e. not independent), making existing models to deal with stratigraphic constraints inadequate.

To overcome these two limitations several solutions have been investigated. Rhodes et al. [15] proposed to tackle limitation B by inferring the age according to Eq. 1 and then by simply ignoring the systematic part of the uncertainty in the analysis of the stratigraphic constraints (using the software Oxcal) [2]. Finally, they used an external criterion to check the coherency of the inference. While pragmatic, this solution does not result in a consistent assessment of uncertainties on ages. Huntriss [11] proposed to tackle limitation A by the following procedure. Two independent samples are generated according to the distributions of $D$ and $\dot{d}$ (assumed to be known). Then their ratio term by term is computed and the resulting sample, distributed according to the distribution of the age $A=D / \dot{d}$, is used to estimate all needed statistics on $A$ (e.g. mean, standard deviation, confidence interval). As we will see it, when the distribution for the annual dosimetry is Gaussian (which is nearly the case), our approach extends this one while dealing with limitation B. Finally, Millard [13] designed a solution to tackle the two limitations. For that purpose, he proposed to model the dosimetry data through a likelihood structure: a latent variable is assigned to each quantity needed for the computation of the dose-rates, these latents (which are typically numerous) relate to observations through Gaussian distributions and to ages through characteristic equivalent doses. Priors are assigned to each latent and the posterior ages are estimated using a Markov Chain Monte Carlo method. The main drawback of this approach is that each dating laboratory may have its own complex protocol to estimate the dose-rate $\dot{d}_{i}$ and for each of these protocols one needs to build a dedicated model that must be implemented, validated (particularly with respect to the choice of the associated priors) and made available to the community. In practice, these factors limit the practical 
impact of such an approach. Moreover, modeling the dosimetry data using observations can be problematic as some parts of the observations and of their associated uncertainties are not strictly speaking associated to observations but rather to expert information (e.g. uncertainty on the water content). Similarly, the systematic component included in the error terms induces a dependence between observations and the associated Bayesian model is, in most cases, not identifiable. Thus the dosimetry data could be considered as a component of the prior structure of the dating model rather than observations. Notice however that for implementation facilities, Millard proposed then to recast the likelihood dependencies into priors (and similarly in a companion article [14]).

In this work, we propose a new solution tackling limitations $\mathrm{A}$ and B. It consists in a Bayesian model for multiplicative multivariate Gaussian errors. Interestingly, this solution is generic with respect to the laboratory protocol as it only relies on the specification of a few variance and covariance values that can be derived for many dose-rate assessment protocols.

The paper is organized as follows. In Section 2.1, we present the age model to estimate an age from an equivalent dose, a dose-rate estimate and its associated standard error. This simple model will be then considered in a multidimensional setting where a part of the dosimetry error is possibly common to several datings (Section 2.2) and where some datings can be constrained to be in a given order (Section 2.3). From that basis, we then consider two situations involving optically stimulated luminescence (OSL) datings $[1,21]$. In a first setting, we consider that we have the raw OSL measurements at our disposal. We thus propose a model consisting in the addition of the age model derived in the previous sections to the Bayesian model, we developed in another paper [7], that infers a characteristic equivalent dose $D$ from Single Aliquot and Regenerative (SAR) dose measurements (Section 3.1). In a second setting, we consider that we only have the output characteristic equivalent doses and their standard errors at our disposal. We thus directly consider their conversions to ages (Section 3.2). Then, we give an application of these two models to infer a chronology from a set of luminescence measurements of five OSL samples with stratigraphic constraints (Section 4). Finally, we summarize our contributions and 
recommendations (Section 5).

\section{Age to equivalent dose relationship: multiplicative Gaussian error}

Hereafter $N\left(\mu, \sigma^{2}\right)$ denotes the Gaussian distribution with mean $\mu$ and variance $\sigma^{2}$.

\subsection{Individual age model}

Once the characteristic equivalent dose $D$ is assessed, the age of the studied sediment since last resetting (corresponding to exposure to light) can be obtained by dividing $D$ by the mean annual dose-rate $\dot{d}$. However in practice, $\dot{d}$ is only known with an uncertainty aggregating many sources of errors due to measurements, calibrations and a low level of awareness of some characteristics of the deposit environment. As a result, even with a precise assessment of the characteristic equivalent dose $D$, the estimated age may be associated to a significant uncertainty. Formally, the relationship between the characteristic equivalent dose and the corresponding characteristic age $A$ is handled by the following multiplicative gaussian error model:

$$
D=A(\dot{d}+\epsilon)
$$

where $\epsilon$ is a Gaussian variable $\epsilon \sim N\left(0, \sigma_{\dot{d}}^{2}\right)$. It results in the following conditional distribution of $D$ given $A$ :

$$
D \sim N\left(A \cdot \dot{d}, A^{2} \cdot \sigma_{\dot{d}}^{2}\right)
$$

We are interested in estimating the age $A>0$ from the observed characteristic equivalent dose $D$, and the dose-rate data $\dot{d}$ and $\sigma_{\dot{d}}$. One can show that the Jeffreys prior for $A$ is

$$
p(A) \propto \frac{1}{A} \mathbb{I}_{[0,+\infty[}(A) .
$$

where $\mathbb{I}$ is the indicator function i.e. for a set $S, \mathbb{I}_{S}(x)=1$ if $x \in S$ and 0 else. This is a particular case of the prior obtained in Appendix A.1. The prior is improper but the resulting posterior is proper for $D \neq 0$ :

$$
P(A \mid D) \propto \frac{1}{A^{2}} \exp \left(-\frac{(A \dot{d}-D)^{2}}{2 A^{2} \sigma_{\dot{d}}^{2}}\right) \mathbb{I}_{[0,+\infty[}(A),
$$


Note that even if $A$ is not a scale parameter for the model defined in Eq. 3, the prior on $A$ is the same as the Jeffreys prior for scale parameter. It is also important to note that if we choose the improper prior $\pi(A) \propto \mathbb{I}_{[0,+\infty[}(A)$, that is the Jeffreys' prior for a location parameter, then the posterior distribution is not defined. Moreover, $A$ is not a location parameter and assigning it to an uniform prior has no objective justification.

Interestingly, the posterior distribution of $A$ defined in Eq. 5 coincides with the distribution of the ratio $D / \mathbf{d}$ where $D \neq 0$ is fixed and where $\mathbf{d}$ is a truncated Gaussian random variable $\mathbf{d} \sim N\left(\dot{d}, \sigma_{\dot{d}}^{2}\right) \cdot \mathbb{I}_{0 ; \infty[}\left(\right.$ where $\mathbb{I}_{] 0 ; \infty[}$ indicates that the density is truncated on $] 0 ; \infty[$ ). To check this fact, we simply apply the change of variables formula. Let $\phi$ be the density of the variable $\mathbf{d}, \phi(\mathbf{d}) \propto \exp \left(-(\mathbf{d}-\dot{d})^{2} /\left(2 \sigma_{\dot{d}}^{2}\right)\right) \mathbb{I}_{] 0 ; \infty[}(\mathbf{d})$, then the density of the variable $A=D / \mathbf{d}$ on $\mathbb{R}^{+}$is

$$
\begin{aligned}
p(A)=\left|\frac{\partial}{\partial A}(D / A)\right| \phi(D / A)=D / A^{2} \phi(D / A) & \propto D /\left(A^{2} \sigma_{\dot{d}}\right) \exp \left(-\frac{(\dot{d}-D / A)^{2}}{2 \sigma_{\dot{d}}^{2}}\right) \\
& \propto \frac{1}{A^{2}} \exp \left(-\frac{(A \dot{d}-D)^{2}}{2 A^{2} \sigma_{\dot{d}}^{2}}\right),
\end{aligned}
$$

which coincides with the distribution given by Eq. 5. So in this specific case, characterizing $A$ consists in simulating $D / \mathbf{d}$ with $\mathbf{d} \sim N\left(\dot{d}, \sigma_{\dot{d}}^{2}\right) \cdot \mathbb{I}_{0 ; \infty}$ As mentioned in the introduction, this simulation based solution has been investigated in a previous work on OSL datings [11].

Note that in practice, the domain of validity of $A$ is limited to a particular period of the history (often called the study period) $\left[a_{\min }, a_{\max }\right]$ with $0<a_{\min }<a_{\max }$. This information can modeled by modifying the prior as follows:

$$
p(A) \propto \frac{1}{A} \mathbb{I}_{\left[a_{\min }, a_{\max }\right]}(A) .
$$

Notice that bounding the support of the prior is motivated by the application, but is also needed to ensure the existence of the posterior moments. In the following synthetic experiments, we fix values for $a_{\min }$ and $a_{\max }$ such that they largely contain the ages of interest: the true ages are chosen in the interval $[100 ; 200]$ while $\left[a_{\min } ; a_{\max }\right]$ is set to $[1 ; 400]$. 
Figure 1 shows how the posterior is modified as a function of $\sigma_{\dot{d}}$. Particularly, when $\sigma_{\dot{d}}$ increases the distribution becomes more asymmetric so that its mode is shifted toward the left while its mean is shifted toward the right. This points out the problem of summary statistics for skewed distributions. The median is favored in this context, because it is invariant by reparametrization (e.g. logarithmic transformation). In a Bayesian context, this question is less crucial since the inference is based on credible regions, as the highest posterior density (HPD) regions, which take into account asymmetry. This also emphasizes the potential error committed using Eq. 1. In practice, the overlap between the 95\% HPD intervals and the corresponding usual intervals $\left[\hat{A}-1.96 \sigma_{A}, \hat{A}+1.96 \sigma_{A}\right]$ (as get using Eq. 1 ) is in average of $97 \%, 95 \%, 91 \%$ and $88 \%$ for the different levels $\sigma_{\dot{d}}=0.05,0.1,0.15,0.2$.

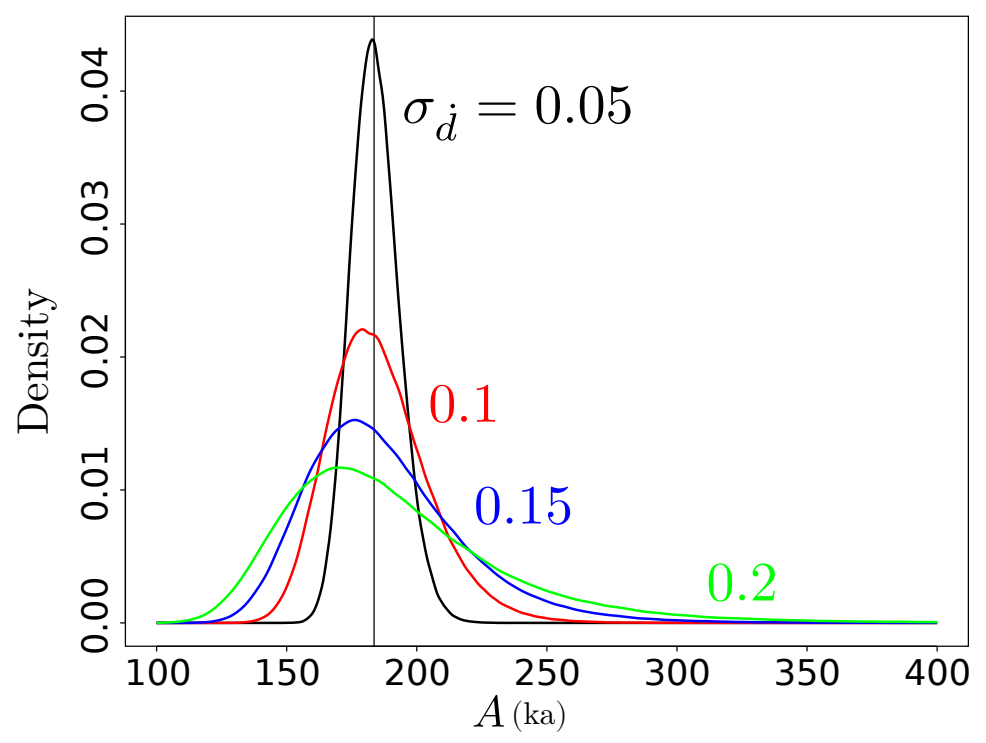

Figure 1: Posterior distribution of $A$ for $\sigma_{\dot{d}}=0.05,0.1,0.15,0.2$ with $\dot{d}=1$. The vertical line represents the true value of $D / \dot{d}$.

\subsection{Multivariate model: sequence of ages}

Let then consider the model for $N$ ages indexed as $A_{i}, i=1, \ldots, N$. For this purpose, we need to explicit the content of $\sigma_{\dot{d}}$. In practice, the dose-rate is estimated as combinations of physical quantities that are known with an uncertainty. A part of this uncertainty is specific to the measured sample (e.g. counting errors due to the spectrometer) and another 
-the systematic error- is related to the measurement device and is common to all datings of the studied sequence (e.g. error on the calibration of the measurements device). Without loss of generality, we will consider in the following a simple case composed of these two sources of error (a more sophisticated instantiation leading to structurally the same model will be considered in Section 4):

$$
D_{i}=A_{i} \cdot \dot{d}_{i}+A_{i} \cdot \epsilon_{\dot{d}, i}+A_{i} \cdot \alpha_{i} \cdot \epsilon_{\dot{d}, c}
$$

where $\epsilon_{\dot{d}, i} \sim N\left(0, \sigma_{\dot{d}, i}^{2}\right)$ is the individual error for sample $i, \epsilon_{\dot{d}, c} \sim N\left(0, \sigma_{\dot{d}, c}^{2}\right)$ is the systematic error and $\alpha_{i}>0$ is known and gives, for each dating $i$, the degree of contamination to the systematic error. Assuming that the noise terms $\epsilon_{\dot{d}, c}$ and $\epsilon_{\dot{d}, i}$ (for all $i$ ) are independent, the conditional distribution of $\left(D_{1}, \cdots, D_{N}\right)$ given $\left(A_{1}, \cdots, A_{N}\right)$ can be written as a Gaussian vector with mean $\left(A_{1} \dot{d}_{1}, \cdots, A_{N} \dot{d}_{N}\right)$ and covariance matrix $\Sigma$ :

$$
\left(D_{1}, \cdots, D_{N}\right) \sim \mathcal{N}\left(\left(A_{1} \dot{d}_{1}, \cdots, A_{N} \dot{d}_{N}\right), \Sigma\right),
$$

where the covariance matrix $\Sigma$ depends on $A_{1}, \cdots, A_{N}$ and writes:

for $i=1, \ldots, N$

$$
\Sigma_{i, i}=A_{i}^{2}\left(\sigma_{\dot{d}, i}^{2}+\alpha_{i}^{2} \sigma_{\dot{d}, c}^{2}\right),
$$

and for $i \neq j$,

$$
\Sigma_{i, j}=A_{i} A_{j} \alpha_{i} \alpha_{j} \sigma_{\dot{d}, c}^{2} .
$$

This covariance matrix is positive definite as long as at least $N$ of the variances $\left(\sigma_{d, i}^{2}\right)_{i=1, N}$ and $\sigma_{d, c}^{2}$ are not equal to zero (assuming that the $\alpha_{i}$ are strictly positive). An example of derivation of a matrix $\Sigma$ from a practical situation is discussed in Section 4.1 and described in Appendix B.

Again, the Jeffreys prior for $\left(A_{1}, \cdots, A_{N}\right)$ writes:

$$
P\left(A_{1}, \cdots, A_{N}\right) \propto \prod_{i=1}^{N}\left(\frac{1}{A_{i}} \mathbb{I}_{\left[a_{\min } ; a_{\max }\right]}\left(A_{i}\right)\right)
$$

The proof is relegated in Appendix A.1. This choice of prior leads to the following posterior distribution:

$$
P\left(A_{1}, \cdots, A_{N} \mid D_{1}, \cdots, D_{N}\right) \propto P\left(D_{1}, \cdots, D_{N} \mid A_{1}, \cdots, A_{N}\right) P\left(A_{1}, \cdots, A_{N}\right),
$$


that will be characterized using a Metropolis-within-Gibbs sampler [16].

Figure 2 shows how the inference on the same age $A_{1}$ is modified when $N$, the number of ages in the sequence, increases. Obviously, the studied model is different from the one in which the inferences on the ages are independent from each others. However, it is satisfactory to observe that the marginal posteriors for a given age are stable when $N$ increases.

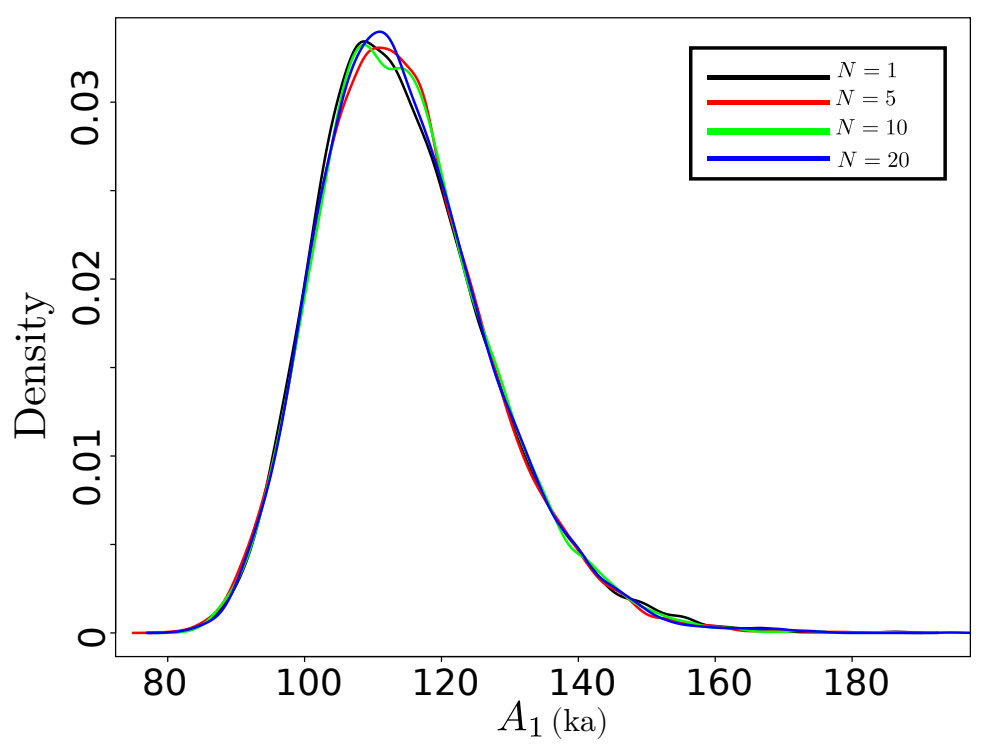

Figure 2: Posterior distribution for the age $A_{1}$ when the number of ages in the sequence increases $(N=1,5,10,20)$. We fix for all $i \sigma_{\dot{d}, i}=\alpha_{i} \sigma_{\dot{d}, c}=0.1$ and the $\dot{d}_{i}$ are randomly draw from a $U(0.5,1.5)$.

\subsection{Sequence of ages with ordering constraints}

In practice, the previous model is of few interest as none of the datings $A_{i}$ interacts directly with the others. Consequently the resulting inference is not so different from what we obtain from a set independent inferences. In this section, we consider the informative case where one knows that the ages are stratigraphically constrained i.e. they (or some of them) are in a known chronological order. This case can be implemented by adding a support constraint for the vector $A_{1}, \cdots, A_{N}$ into its prior [6]. For that purpose, we consider $\mathcal{B}$, the set of $N$-tuples satisfying the imposed stratigraphy (that can be incompletely 
stated i.e. some of the ages can be not fully ordered) and state:

$$
p\left(A_{1}, \cdots, A_{N}\right) \propto \prod_{i=1}^{N}\left(\frac{1}{A_{i}}\right) \mathbb{I}_{\mathcal{B}}\left(A_{1}, \cdots, A_{N}\right),
$$

where $\mathbb{I}_{\mathcal{B}}\left(A_{1}, \cdots, A_{N}\right)=1$ if $\left(A_{1}, \cdots, A_{N}\right) \in \mathcal{B}$ and 0 else.

Moreover, it is possible to allow some of the datings of the sequence not to be affected by the systematic errors (or by different systematic terms). In practice, this situation typically happens when some of the datings are performed by different laboratories making the errors on the assessment of the dose-rates $\dot{d}_{i}$ independent from each others or when some other unrelated dating methods (e.g. radiocarbon dating) are used. Here for a sake of simplicity, we will consider the first case. In practice, it implies to set to zero the covariance terms of $\Sigma$ relating two ages/dosimetries being estimated by two different laboratories.

Let first consider the case where there is no systematic-error free dating. In the experiments displayed in Figures 3 and 4, we used $\sigma_{d, i}=0.1, \alpha_{i} \sigma_{d, c}=0.1$ and $\left(d_{i}\right)_{i}$ randomly draw from a $U(0.5,1.5)$. Figure 3 shows how the error of estimation for a given age is modified when the number of stratigraphically constrained ages increases. Particularly, one observes that the mean and the variance errors decrease slowly with the number of observations. This observation is related to the systematic error term that cannot be inferred without additional information. Figure 4 shows typical posteriors on a given age with an increasing number of stratigraphically constrained ages.

Let then consider the effect of systematic-error free datings. In the experiments displayed in Figures 5 and 6, we consider three settings: in the first one, we consider five datings without any stratigraphic constraints, all affected by the systematic error term. In the second one, we consider the same five datings with stratigraphic constraints (again, all affected by the systematic error term). In the third one, we consider that two of them are not affected by the systematic-error term (while keeping the same overall variance level). Figure 5 shows how the error of estimation for a given age is modified for these different settings. Particularly, one observes that whereas the number of datings is the same in all cases and whereas the overall uncertainty level is similar, the estimation error is, as expected, highly reduced when adding independent datings. Figure 6 shows typical pos- 


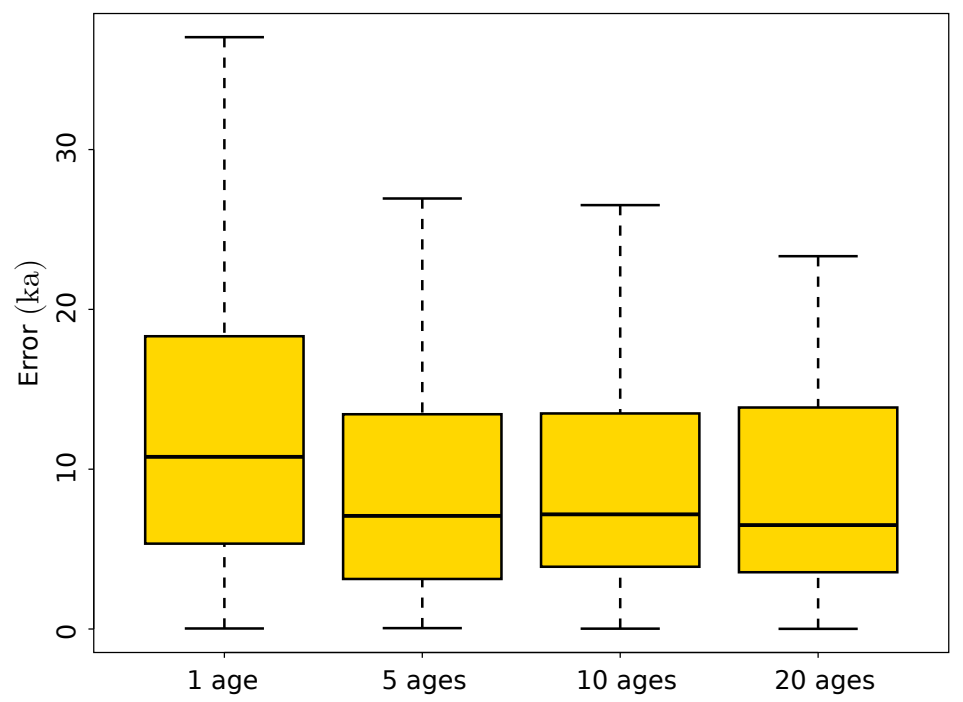

Figure 3: Box plots of the estimation error for a given age when the number of ages in the sequence increases $(N=1,5,10,20)$ over a set of $\mathbf{1 0 0}$ realizations. From left to right, statistics on the 100 experiments for 1, 5, 10 and 20 stratigraphically constrained ages.

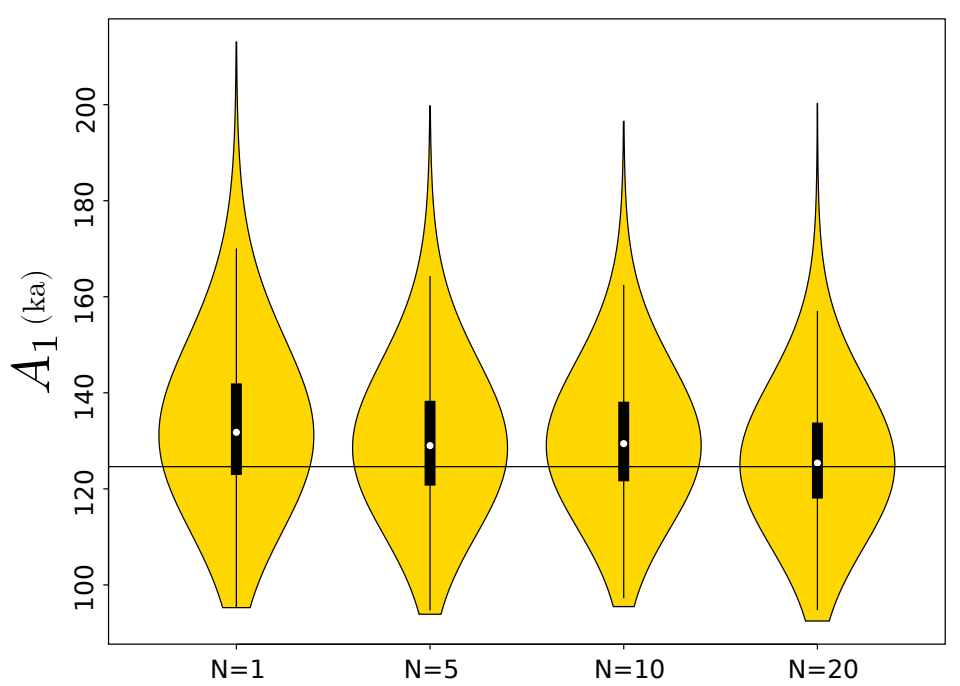

Figure 4: Violin plots of the posterior samples for a given age when the number of ages in the sequence increases $(N=1,5,10,20)$. The horizontal line is the true value of $A_{1}$.

teriors from this set of experiments. It illustrates how the posterior variance diminishes when introducing systematic-error free datings. 


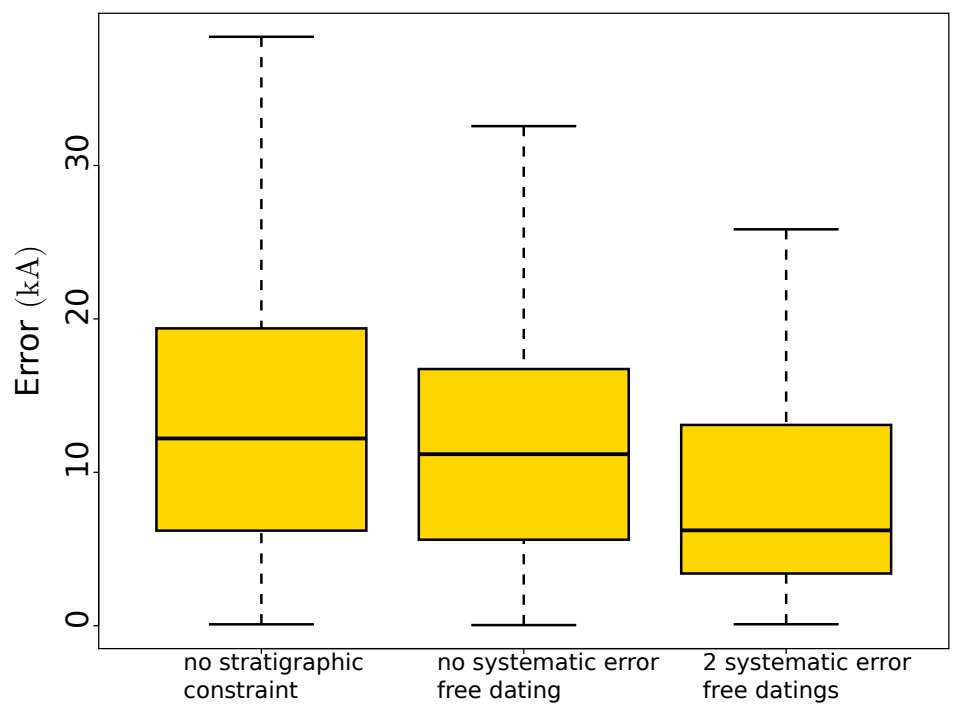

Figure 5: Box plots of the estimation error on the same age $A_{3}$ from a set of 100 realizations. From left to right, statistics on the 100 experiments for 5 ages no stratigraphically constrained, 5 stratigraphically constrained ages and 5 stratigraphically constrained ages whose two are free of systematic error.
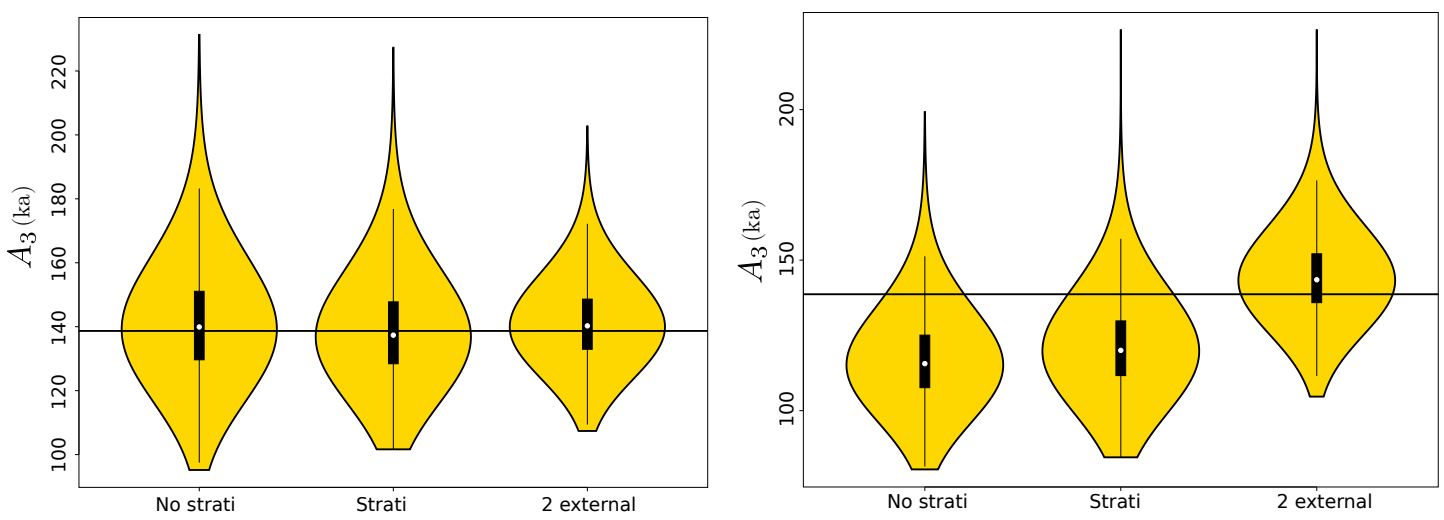

Figure 6: Violin plots of the posterior samples for $A_{3}$ for $N=5$ ages for two typical cases: one where the systematic component of the error is low (left plot) and one where it is high (right plot). The left density is the posterior without stratigraphic constraint, the middle density is the posterior with the stratigraphic constraints and only correlated ages and the right density, the posterior with the stratigraphic constraints but with two systematic-error free datings among the five. The horizontal line is the true value for $A_{3}$. 


\section{Two models for OSL dating}

In this section, we consider the prior we developed in the previous section as a component of a full optically stimulated luminescence (OSL) dating model (Section 3.1). Then, we consider a two-steps approach consisting in assessing the posterior ages $\left(A_{1}, \cdots, A_{N}\right)$ from characteristic equivalent doses estimates and their standard errors $\left(\tilde{D}_{i}, \sigma_{D, i}\right)$ that are computed separately (Section 3.2).

\subsection{Full model}

The model presented in this section is the combination of the age model developed below and of a model for the estimation of characteristic equivalent doses $\left(D_{1}, \cdots, D_{N}\right)$

from normalized luminescence observations (denoted as $\left.\left(N_{i}^{k, j}\right)_{i, j, k},\left(\sigma_{N_{i}^{k, j}}\right)_{i, j, k}\right)$ presented elsewhere [7]. In practice, it simply consists in adding the prior structure

$$
P\left(D_{1}, \cdots, D_{N} \mid A_{1}, \cdots, A_{N}\right) P\left(A_{1}, \cdots, A_{N}\right)
$$

as defined in Eq. 8 and 13 to the marginal model $P\left(D_{1}, \cdots, D_{N} \mid\left(N_{i}^{k, j}\right)_{i, j, k},\left(\sigma_{N_{i}^{k, j}}\right)_{i, j, k}\right)$. The directed acyclic graph (DAG) representing the overall 4-stages hierarchical model is given in Figure 7. Notations are introduced in the caption (see the corresponding paper [7] for more details, the notations have been a bit recast for simplicity). For a sake of completeness, we also recall here the corresponding posterior distribution (removing the exogenous parameters $\dot{d}_{l a b}, t_{i}^{k, j}, \dot{d}_{i}, \sigma_{\dot{d}, i}, \sigma_{\dot{d}, c}, \alpha_{i}$ from the notations for a sake of simplicity):

$$
\begin{aligned}
& P\left(A_{1}, \cdots, A_{N}, D_{1}, \cdots, D_{N},\left(\sigma_{D_{i}}\right)_{i},\left(D_{i}^{1, j}\right)_{i, j},\left(\sigma_{f, i}^{j}\right)_{i, j},\left(\theta_{i}^{j}\right)_{i, j},\left(Q_{i}^{k, j}\right)_{i, j, k} \mid\left(N_{i}^{k, j}\right)_{i, j, k},\left(\sigma_{N_{i}^{k, j}}\right)_{i, j, k}\right) \\
\propto & \prod_{i, k, j}\left(P\left(N_{i}^{k, j} \mid Q_{i}^{k, j}, \sigma_{N_{i}^{k, j}}\right)\right) \\
& \times \prod_{i, k, j}\left(P\left(Q_{i}^{k, j} \mid \sigma_{f, i}^{j}, \theta_{i}^{j}, D_{i}^{1, j}\right)\right) \prod_{i, j}\left(P\left(D_{i}^{1, j} \mid D_{i}, \sigma_{D, i}\right) P\left(\sigma_{D, i} \mid D_{i}\right) P\left(\sigma_{f, i}^{j}\right) P\left(\theta_{i}^{j}\right)\right) \\
& \times P\left(D_{1}, \cdots, D_{N} \mid A_{1}, \cdots, A_{N}\right) P\left(A_{1}, \cdots, A_{N}\right),
\end{aligned}
$$


with the age model $P\left(D_{1}, \cdots, D_{N} \mid A_{1}, \cdots, A_{N}\right) P\left(A_{1}, \cdots, A_{N}\right)$ as defined in Eq. 8 and 13 . The other densities (discussed in our previous paper) write:

$$
\begin{aligned}
& P\left(N_{i}^{k, j} \mid Q_{i}^{k, j}, \sigma_{N_{i}^{k, j}}\right) \propto \exp -\frac{\left(N_{i}^{k, j}-Q_{i}^{k, j}\right)^{2}}{2 \sigma_{N_{i}^{k, j}}^{2}} \\
& P\left(Q_{i}^{k, j} \mid \sigma_{f, i}^{j}, \theta_{i}^{j}, D_{i}^{1, j}\right) \propto \frac{1}{\sqrt{2 \pi} \sigma_{f, i}^{j}} \exp -\frac{\left(f_{\theta_{i}^{j}}\left(D_{i}^{k, j}\right)-Q_{i}^{k, j}\right)^{2}}{2 \sigma_{f, i}^{j}{ }^{2}}, \text { with } D_{i}^{k, j}=t_{i}^{k, j} \cdot \dot{d}_{l a b} \text { for } k \geq 2 \\
& P\left(\sigma_{f, i}^{j}\right) \propto e^{-20 \sigma_{f, i}^{j}} \mathbb{I} \mathbb{R}^{+}\left(\sigma_{f, i}\right), P\left(\theta_{i}^{j}=\left[u_{i}^{j}, v_{i}^{j}, w_{i}^{j}, x_{i}^{j}\right]\right) \propto \exp \left(\frac{\left(u_{i}^{j}-6.5\right)^{2}}{170}\right) \mathbb{I} \mathbb{R}^{+}\left(u^{j}\right) \\
& \exp \left(\frac{\left(v_{i}^{j}-50\right)^{2}}{10^{6}}\right) \mathbb{I} \mathbb{R}^{+}\left(v^{j}\right) \exp \left(\frac{\left(w_{i}^{j}-0.002\right)^{2}}{0.0002}\right) \mathbb{I} \mathbb{R}^{+}\left(w^{j}\right) \exp \left(\frac{\left(z_{i}^{j}-0.5\right)^{2}}{12.5}\right) \mathbb{I}_{\left[-u^{j}, \infty[\right.}\left(z^{j}\right), \\
& P\left(D_{i}^{1, j} \mid D_{i}, \sigma_{D, i}\right) \propto\left[\frac{\sigma_{D_{i}}}{\left(D_{i}^{1, j}-D_{i}\right)^{2}+\sigma_{D_{i}}^{2}}\right], P\left(\sigma_{D, i} \mid D_{i}\right)=\frac{0.16 D_{i}}{\sigma_{D, i}^{2}+\left(0.16 D_{i}\right)^{2}} \mathbb{I} \mathbb{R}^{+}\left(\sigma_{D, i}\right) .
\end{aligned}
$$

The inference is then performed using a Metropolis-within-Gibbs sampler. In particular, we can provide estimates for the ages $A_{1}, \cdots, A_{N}$ from the normalized luminescence measurements $\left(N_{i}^{k, j}\right)_{i, j, k}$, their associated standard deviations $\left(\sigma_{N_{i}^{k, j}}\right)_{i, j, k}$ and the dosimetry data $\dot{d}_{i}, \sigma_{\dot{d}, i}, \sigma_{\dot{d}, c}$ and $\alpha_{i}$. Some results will be discussed in Section 4 .

\subsection{Two-steps model}

In this section, we consider that we want to infer a set of ages from a set of characteristic equivalent doses estimates $\tilde{D}_{i}$, their associated standard errors $\sigma_{D, i}$, the dose-rate estimates and standard errors and a set of stratigraphic constraints. This situation typically arises when the chronological scenario is built using several pool of datings published by other research groups. In such a case, the high-level results (such as the $\tilde{D}_{i}, \sigma_{D, i}$ ) are commonly given in the corresponding publications but most of the times the raw luminescence data are not available. Moreover, such an approach allows users of other non-Bayesian approaches to estimate the $\tilde{D}_{i}, \sigma_{D, i}$ (see $[9,8]$ ) to account properly for ordering constraints on their data. 


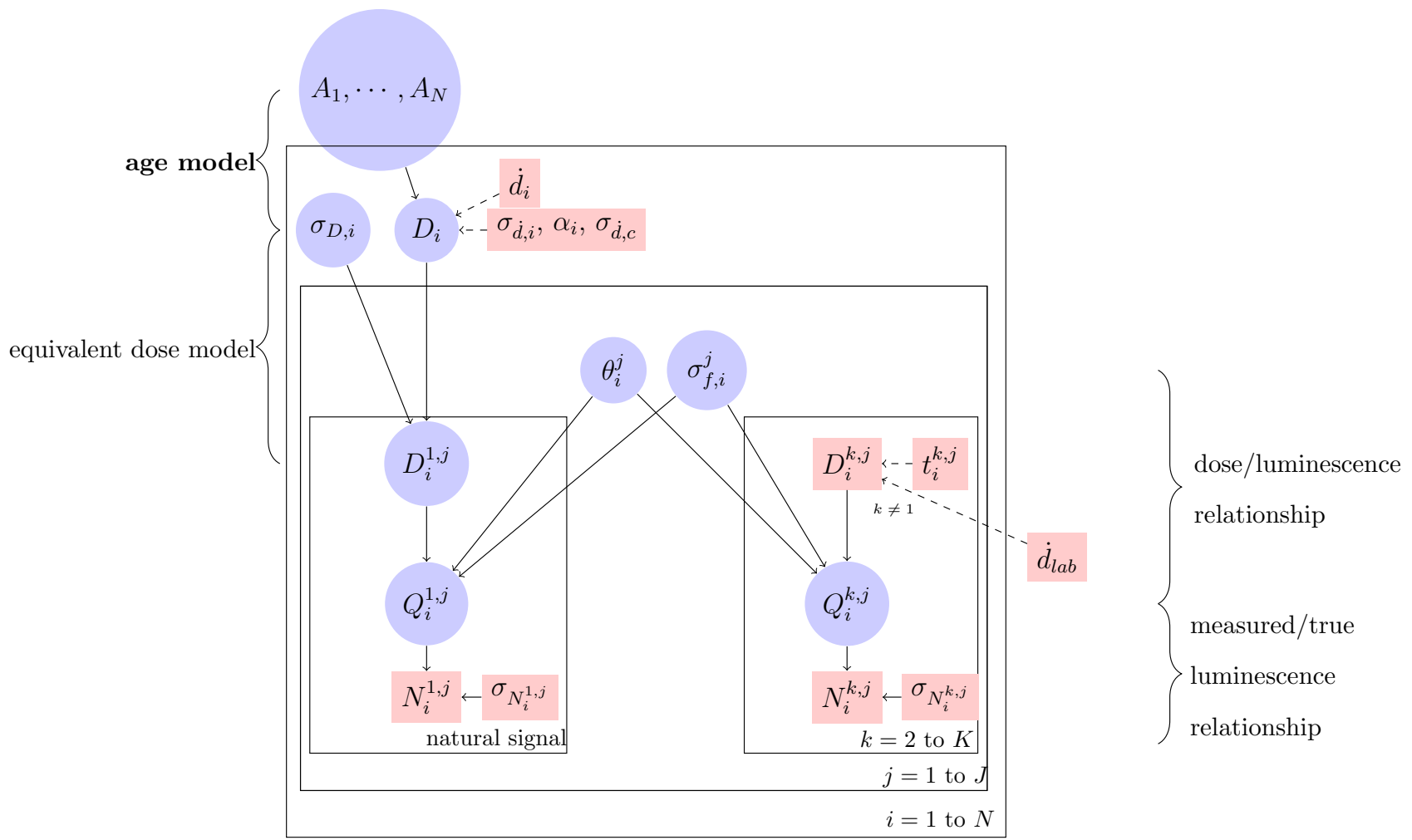

Figure 7: Directed acyclic graph (DAG) of the inference model. Sequence level: The vector of ages $A_{1}, \cdots, A_{N}$ is related to the vector of characteristic equivalent doses $D_{1}, \cdots, D_{N}$ by the age model taking into account for the different kinds of uncertainties on the dosimetry. Sample $i$ level: For each sample $i$, the characteristic equivalent dose $D_{i}$ and dispersion $\sigma_{D, i}$ are common to all aliquots of the sample. Aliquot $j$ level: Each aliquot $j$ is associated with a natural dose $D_{i}^{1, j}$, a set of regenerative dose $D_{i}^{k, j}(k \geq 2)$ and a dose response function $f_{\theta_{i}^{j}}$ with its standard error $\sigma_{f, i}^{j}$. Dose $k$ level: This function links the natural and regenerative doses $D_{i}^{k, j}$ and the true normalized signal $Q_{i}^{k, j}$ while each of this signal is related the corresponding observation $N_{i}^{k, j}$. Plain arrows represent stochastic relationships, dashed arrows represent deterministic relationships, blue circles represent model parameters and red boxes represent observations and exogenous parameters. In this DAG, we represented a single aliquot $j$ and a single sample $i$ level but they may be numerous.

\subsubsection{Individual age model}

As for the previous model, the ages are related to the true characteristic equivalent doses $D_{i}$ through:

$$
D_{i}=A_{i} \dot{d}_{i}+A_{i} \epsilon_{\dot{d}, i}
$$


which are themselves related to the inferred/measured characteristic equivalent dose $\tilde{D}_{i}$ by:

$$
\tilde{D}_{i}=D_{i}+\epsilon_{D, i}
$$

where $\epsilon_{\dot{d}, i} \sim N\left(0, \sigma_{\dot{d}, i}^{2}\right)$ and $\epsilon_{D, i} \sim N\left(0, \sigma_{D, i}^{2}\right)\left(\sigma_{D, i}\right.$ being the standard error associated to $\left.\tilde{D}_{i}\right)$ are independent. This results in:

$$
\tilde{D}_{i} \mid A_{i} \sim \mathcal{N}\left(A_{i} \cdot \dot{d}_{i}, A_{i}^{2} \cdot \sigma_{\dot{d}, i}^{2}+\sigma_{D, i}^{2}\right)
$$

For this specific model, we obtain the Jeffreys prior (proof in Appendix A.2):

$$
p\left(A_{i}\right) \propto \sqrt{\frac{A_{i}^{2}\left(2 \sigma_{\dot{d}, i}^{4}+\dot{d}_{i}^{2} \sigma_{\dot{d}, i}^{2}\right)+\sigma_{D, i}^{2} \dot{d}_{i}^{2}}{\left(A_{i}^{2} \sigma_{\dot{d}, i}^{2}+\sigma_{D, i}^{2}\right)^{2}}} \text { for } 0<a_{\text {min }}<A_{i}<a_{\max } \text { and } 0 \text { else. }
$$

When $\sigma_{D, i}=0$, the graph of this function is equivalent (up to a multiplicative constant) to the one of $A_{i} \mapsto 1 / A_{i}$. When $\sigma_{D, i}^{2}>2 \sigma_{\dot{d}, i}^{2}$ (which will be the case for our application), the graph is strictly decreasing.

\subsubsection{Multidimensional model : sequence of ages}

In this section, we consider a multidimensional extension of the model presented below analogously to what we performed in Section 2. For that purpose, we modified Eq. 14 as

$$
D_{i}=A_{i} \dot{d}_{i}+A_{i} \epsilon_{\dot{d}, i}+A_{i} \alpha_{i} \epsilon_{\dot{d}, c}
$$

where $\epsilon_{\dot{d}, i} \sim N\left(0, \sigma_{\dot{d}, i}^{2}\right), \epsilon_{D, i} \sim N\left(0, \sigma_{D, i}^{2}\right)$ and $\epsilon_{\dot{d}, c} \sim N\left(0, \sigma_{\dot{d}, c}^{2}\right)$ are independent and where $\alpha_{i}>0$ known gives, for each dating $i$, the degree of contamination to the systematic error. This results in:

$$
\tilde{D}_{1}, \cdots \tilde{D}_{N} \mid A_{1}, \cdots, A_{N} \sim \mathcal{N}\left(\left(A_{i} \cdot \dot{d}_{i}\right)_{i}, \Sigma+I_{\sigma_{D, i}^{2}}\right),
$$

where $I_{\sigma_{D, i}^{2}}$ is the diagonal matrix such that $I_{i, i}=\sigma_{D, i}^{2}$ and $\Sigma$ built as in Eq. 9 and 10 . Then to be consistent with Eq. 17, we set the prior:

$$
P\left(A_{1}, \cdots, A_{N}\right) \propto \prod_{i=1}^{N} \sqrt{\frac{A_{i}^{2}\left(2\left(\sigma_{\dot{d}, i}^{2}+\alpha_{i}^{2} \sigma_{\dot{d}, c}^{2}\right)^{2}+\dot{d}_{i}^{2}\left(\sigma_{\dot{d}, i}^{2}+\alpha_{i}^{2} \sigma_{\dot{d}, c}^{2}\right)\right)+\sigma_{D, i}^{2} \dot{d}_{i}^{2}}{\left(A_{i}^{2}\left(\sigma_{\dot{d}, i}^{2}+\alpha_{i}^{2} \sigma_{\dot{d}, c}^{2}\right)+\sigma_{D, i}^{2}\right)^{2}}} \mathbb{I}_{\mathcal{B}}\left(A_{1}, \cdots, A_{N}\right),
$$


we can then write the posterior:

$$
P\left(A_{1}, \cdots, A_{N} \mid \tilde{D}_{1}, \cdots \tilde{D}_{N}\right) \propto P\left(\tilde{D}_{1}, \cdots \tilde{D}_{N} \mid A_{1}, \cdots, A_{N}\right) P\left(A_{1}, \cdots, A_{N}\right),
$$

that can be characterized using a Metropolis-within-Gibbs sampler. In particular, we can provide estimates for the ages $A_{1}, \cdots, A_{N}$ from a set of inferred/measured characteristic equivalent doses $\tilde{D}_{i}$, their associated standard errors $\sigma_{D, i}$ and the dosimetry data $\dot{d}_{i}, \sigma_{\dot{d}, i}, \sigma_{\dot{d}, c}$ and $\alpha_{i}$. Some results will be discussed in Section 4 . 


\section{Application}

In this section, we first present a set of real measurements associated to five OSL samples stratigraphically constrained [19]. Then, we use the two methods presented in Section 3.1 and 3.2 to process these measurements.

\subsection{Data and parameters}

The five studied sediments respectively called OSL1, OSL4, OSL6, OSL7 and OSL8 (we kept the names given in the original publication) have been sampled from a sedimentary pile corresponding to the Middle Stone Age. This leads to the following upper and lower bound for the ages: $a_{\min }=25 \mathrm{ka}$ and $a_{\max }=280 \mathrm{ka}$. The stratigraphy imposes the ages to be ordered such that $A_{1} \geq A_{4} \geq A_{6} \geq A_{7} \geq A_{8}$. Details on the measurements protocol can be found in the original paper [19]. In the following, we focus on the way the dose-rate

$\dot{d}_{i}$ is characterized for this specific example. The dose-rate $\dot{d}_{i}$ is considered as the sum of its $\beta, \gamma$ and cosmic components (the $\alpha$ component is supposed to be negligible):

$$
\dot{d}_{i}=\dot{d}_{\beta, i}+\dot{d}_{\gamma, i}+\dot{d}_{c o s, i}
$$

In practice, these three quantities are estimated through the combination of several physical quantities that are themselves not completely known. More specifically, for each sample $i$, one assesses values for the uranium, thorium and potassium contents $U_{i}, T h_{i}$ and $K_{i}$, the gamma radiation component $\gamma_{i}$, the cosmic radiation component $\cos _{i}$, the saturation uptake content $W_{i}$ and the fraction of average water content $F_{i}$. These six quantities are subject to systematic and independent errors (that are detailed in Appendix B). The corresponding error model relating these quantities to the total dose-rate $\dot{d}_{i}$ is then linearized to get a sum of zero-mean gaussian error terms that fully define the covariance matrix $\Sigma$ involved in our multivariate equivalent dose to age model (Eq. 8, 9 and 10). The resulting values for the studied sediments are given in Table 1 . The derivation needed to get them are close to the ones used in practice to get $\mu_{\dot{d}}$ and $\sigma_{\dot{d}}$ in the standard approach (Eq. 1) and are detailed in Appendix B. 


\begin{tabular}{|c|c|c|c|c|c|}
\hline & OSL8 & OSL7 & OSL6 & OSL4 & OSL1 \\
\hline$\dot{d}_{i}(\mathrm{~Gy})$ & 1.40 & 1.30 & 1.25 & 1.33 & 1.13 \\
\hline$\sigma_{\dot{d}, i}(\mathrm{~Gy})$ & 0.03 & 0.03 & 0.03 & 0.03 & 0.03 \\
\hline$\alpha_{\dot{d}, i} \sigma_{\dot{d}, c}(\mathrm{~Gy})$ & 0.08 & 0.09 & 0.09 & 0.09 & 0.10 \\
\hline
\end{tabular}

Table 1: Summary of the quantities defining $\Sigma$ for the five studied samples.

Another source of uncertainty that is commonly considered to estimate an age from an equivalent dose is the uncertainty on the laboratory source used for administering the regenerative and test doses. This source is only known with a calibration error of known standard error $\sigma_{l a b}$ (which, considering that all the samples have been processed using the same experimental device, is a systematic term). The associated error is often called a percent error and impacts $D_{i}$ as a percentage of itself [1]. A convenient way to take into account for this supplemental source of uncertainty consists in simply modifying the covariance matrix as:

$$
\begin{aligned}
\Sigma_{i, i} & =A_{i}^{2}\left(\sigma_{\dot{d}, i}^{2}+\alpha_{i}^{2} \sigma_{\dot{d}, c}^{2}+\dot{d}_{i}^{2} \sigma_{l a b}^{2}\right), \\
\text { for } i \neq j, \Sigma_{i, j} & =A_{i} A_{j}\left(\alpha_{i} \alpha_{j} \sigma_{\dot{d}, c}^{2}+\dot{d}_{i} \dot{d}_{j} \sigma_{l a b}^{2}\right) .
\end{aligned}
$$

Here we set $\sigma_{l a b}=0.05$.

\subsection{Results}

\subsubsection{Preliminary}

In this section, we study the full model of Section 3.1 without the stratigraphic constraints between the five samples. As for the simple model of Section 2.2, without constraint, we expect the age model not to affect the overall inference and particularly the posteriors on the characteristic equivalent doses $D_{i}$. Figure 8 displays the posterior distribution for a given age when using one and five ages (left side) and for a given characteristic equivalent dose when using no age model, the age model with one age and the age model with the five ages (right side). One can observe that, as for the non-hierarchical model, the age and equivalent dose posteriors remain sensibly unchanged. 

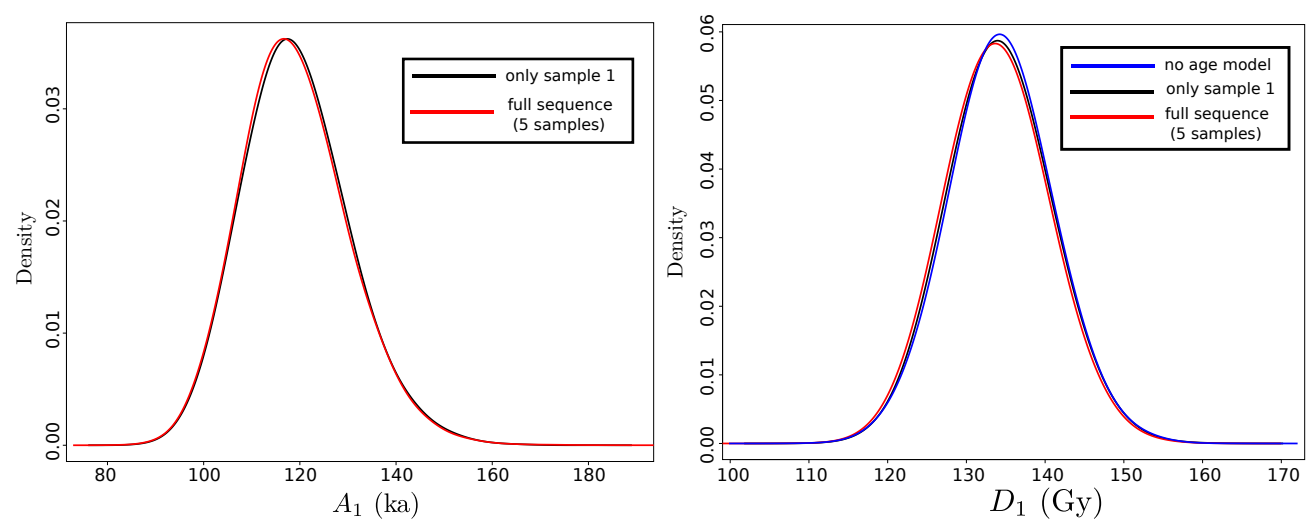

Figure 8: Marginalized posterior densities for the age $A_{1}$ (left) and for the characteristic equivalent dose $D_{1}$ (right) without stratigraphic constraint. The black curves are the posteriors estimated using a single age and the red ones are the posteriors estimated using the five ages (without stratigraphic constraint). The characteristic equivalent dose obtained when considering only the characteristic equivalent dose model is displayed in blue.

\subsubsection{Comparison of the approaches}

Figure 9 displays the posterior for each age of the sequence with and without imposing the stratigraphic constraints. Table 2 gives a summary of the inferences obtained using the full-model and the two-steps model (using the characteristic equivalent dose posteriors as inputs) when considering the five data-sets together or when studying them one by one (i.e. without the stratigraphic constraints). The ages obtained in the original publication [19] with a classical approach are also given. First, note that the estimated equivalent doses and ages (without the stratigraphic constraints) are notably higher than those obtained in the original publication. This point is not related to the age model and has been already noticed and analyzed on a set of controlled data [10] where characteristic equivalent doses obtained using the Bayesian approach [7] have been observed to be more consistent with associated external datings than those obtained using a standard approach. Secondly, the results of the comparison can be summarized as:

- the ages have been made internally consistent with the stratigraphy,

- the posterior standard deviation is slightly reduced when using the stratigraphic constraints, 
- the full and the two-steps models give similar inferences for the ages.

This last point is an interesting result suggesting that the two-steps method can be used with confidence when the OSL measurement data are not available. However this conclusion is related to the fact that the equivalent doses are estimated by the same Bayesian model in both situations. If the data $\left(\tilde{D}_{i}, \sigma_{D, i}\right)_{i}$ are estimated using an other equivalent dose model (e.g. the minimal age model or the finite mixture model), we cannot expect both our full model and two-step model to match well. The two-step model will be strongly affected by the quality of data $\left(\tilde{D}_{i} \sigma_{D, i}\right)_{i}$, depending on the choice of the estimate.

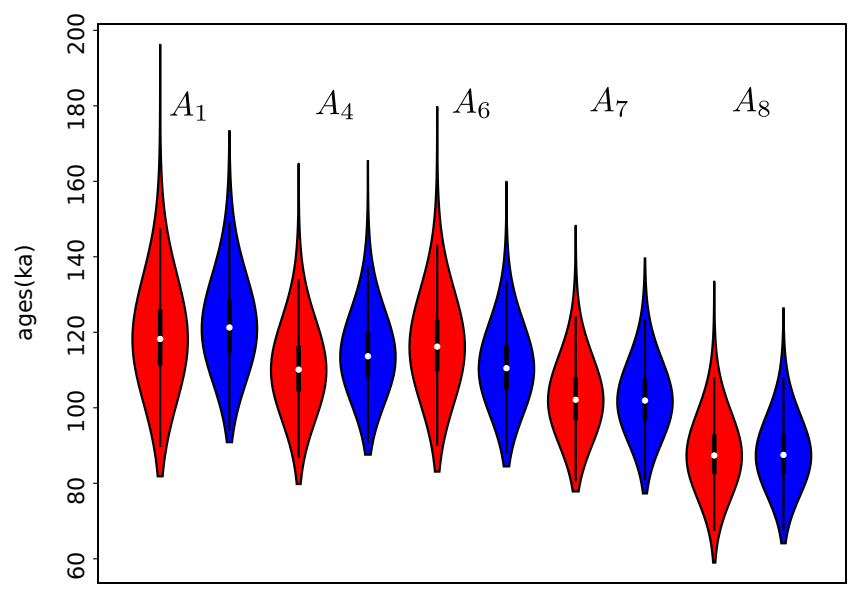

Figure 9: Violin plots from the marginalized posterior densities for the ages with stratigraphic constraints (blue) and without (red). 


\begin{tabular}{|c||c|c|c|c|c|c|}
\hline & $\cdot$ & $A_{8}$ & $A_{7}$ & $A_{6}$ & $A_{4}$ & $A_{1}$ \\
\hline \multirow{3}{*}{ no strati } & full & $88.03 \pm 7.78$ & $102.79 \pm 8.32$ & $117.38 \pm 10.14$ & $110.68 \pm 9.09$ & $118.86 \pm 11.01$ \\
\cline { 2 - 7 } & two-steps & $88.19 \pm 7.82$ & $102.03 \pm 8.22$ & $117.92 \pm 10.07$ & $110.86 \pm 9.24$ & $119.67 \pm 11.05$ \\
\hline \hline \multirow{2}{*}{ strati } & full & $88.05 \pm 7.62$ & $102.43 \pm 8.03$ & $111.12 \pm 8.65$ & $114.31 \pm 8.87$ & $122.10 \pm 10.46$ \\
\cline { 2 - 7 } & two-steps & $88.45 \pm 7.78$ & $102.30 \pm 8.17$ & $111.99 \pm 8.95$ & $115.39 \pm 9.25$ & $123.01 \pm 10.65$ \\
\hline \hline \multicolumn{2}{|c|}{ Original estimates [19] } & $83 \pm 8$ & $89 \pm 8$ & $109 \pm 10$ & $100 \pm 10$ & $100 \pm 10$ \\
\hline
\end{tabular}

Table 2: Four first lines: Posterior means and standard deviations for the five ages for the full and the two-steps model with and without the stratigraphic constraints. Last line: Estimates as given in [19]. 


\section{Conclusion}

In this work, we considered a multivariate model with multiplicative Gaussian error in a Bayesian framework. This model was then used as a key component of OSL dating models from normalized luminescence data or from characteristic equivalent dose estimates. As mentioned in introduction, such a multiplicative model is not specific to OSL but related to any paleo-dosimetric dating method (e.g. Thermo-luminescence (TL), Electron Spin Resonance (ESR), Infrared stimulated luminescence (IRSL) datings) and we hope this work will be also useful to these approaches. Notice that interestingly, based on a more intuitive reasoning than the derivation we provide here, the use of the $1 / A$ prior has already been evoked by Millard [14] in a general discussion about prior distributions for ages and dates in chronometric modeling.

The two proposed dating models were then applied to a simple real dataset to illustrate the interest of including stratigraphic constraints when available. The inferences given by the full and the two-steps approaches are similar on this example. This is a positive result suggesting that the two-steps method can be used when the measurement data are not available. Notice that the dataset we used is unfortunately free of external dating allowing to correct the systematic components of the error. Datasets including such external datings (e.g. [15]) would benefit greatly from these models. The two-steps model and the full model will be made available in a dedicated software to allow users to incorporate OSL datings in addition to other datings to build chronologies and to explore deeply their differences.

To our opinion, the main limitation of our approach consists on the assumption of gaussianity of the distributions associated to the dosimetry errors. While there is, strictly speaking, no reason to assume a gaussian shape for the dosimetry error, we think that this limitation is in practice a minor point. First, one can visualize the true error model on the dosimetry errors by simulation and then check that a gaussian shape is a good approximation (which has been observed to be the case [11, 22] and which is, in most cases, performed in the standard approach). Second, one must remind here that some error terms involved in the dose rate estimation are not well characterized and that in 
practice, only coarse (and generally highly conservative [15]) estimations are available.

\section{References}

[1] J. Aitken. Introduction to Optical Dating: The Dating of Quaternary Sediments by the Use of Photon-stimulated Luminescence. Clarendon Press, 1998.

[2] C. Bronk Ramsey. Radiocarbon calibration and analysis of stratigraphy: the oxcal program. Radiocarbon, 37(2):425-430, 1995.

[3] C. Bronk Ramsey. Bayesian analysis of radiocarbon dates. Radiocarbon, 51(1):337$360,2009$.

[4] C. Buck, J. Christen, and G. James. Bcal : an on-line Bayesian radiocarbon calibration tool. Internet Archaeology, 7, 1999.

[5] C Buck, J Kenworthy, C Litton, and A Smith. Combining archaeological and radiocarbon information: a Bayesian approach to calibration. Antiquity, 65(249):808-821, 1991.

[6] C.E. Buck and W.G. Cavanagh and C. Litton C. Bayesian Approach to Intrepreting Archaeological Data. Wiley, 1996.

[7] B. Combès, A. Philippe, P. Lanos, N. Mercier, C. Tribolo, G. Guerin, P. Guibert, and C. Lahaye. A Bayesian central equivalent dose model for optically stimulated luminescence dating. Quaternary geochronology, 28:62-70, 2015.

[8] G.A.T. Duller. Assessing the error on equivalent dose estimates derived from single aliquot regenerative dose measurements. Ancient TL, 25:15-24, 2007.

[9] R. F. Galbraith, R. G. Roberts, G. M. Laslett, H. Yoshida, and J. M. Olley. Optical dating of single and multiple grains of quartz from jinmium rock shelter, northern australia: Part 1, experimental design and statistical models. Archaeometry, 41(2):339364, 1999. 
[10] G. Guérin, B. Combès, C. Lahaye, K. J. Thomsen, C. Tribolo, P. Urbanova, P. Guibert, N. Mercier, and H. Valladas. Testing the accuracy of a bayesian central-dose model for single-grain osl, using known-age samples. Radiation Measurements, 81:62-70, 2015.

[11] A. Huntriss. A Bayesian analysis of luminescence dating. PhD thesis, University of Durham, UK, 2007.

[12] Ph. Lanos, A. Philippe, H. Lanos, and Ph Dufresne. Chronomodel : Chronological modelling of archaeological data using Bayesian statistics. http://www . chronomodel. fr, 2015.

[13] A.R. Millard. Bayesian analysis of ESR dates, with application to Border Cave. Quaternary Geochronology, 1(2):159 - 166, 2006.

[14] A.R. Millard. Bayesian analysis of pleistocene chronometric methods. Archaeometry, 48(2):359-375, May 2006.

[15] E.J. Rhodes, C. Bronk Ramsey, Z. Outram, C. Batt, L. Willis, S. Dockrill, and J. Bond. Bayesian methods applied to the interpretation of multiple OSL dates: high precision sediment ages from old scatness broch excavations, shetland isles. Quaternary Science Reviews, 22(1013):1231 - 1244, 2003.

[16] G.O. Roberts and J.S. Rosenthal. Examples of adaptive MCMC. Journal of Computational and Graphical Statistics, 18(2):349-367, 2009.

[17] J.R. Taylor. An Introduction to Error Analysis: The Study of Uncertainties in Physical Measurements. A series of books in physics. University Science Books, 1997.

[18] R.E. Taylor and M.J. Aitken. Chronometric Dating in Archaeology. Advances in Archaeological and Museum Science. Springer, 1997.

[19] C. Tribolo, N. Mercier, E. Douville, J.-L. Joron, J.-L. Reyss, D. Rufer, N. Cantin, Y. Lefrais, C.E. Miller, G. Porraz, J. Parkington, J.-P. Rigaud, and P.-J. Texier. OSL 
and TL dating of the middle stone age sequence at diepkloof rock shelter (South Africa): a clarification. Journal of Archaeological Science, 40(9):3401 - 3411, 2013.

[20] M-A Vibet, A. Philippe, Ph. Lanos, and Ph Dufresne. Chronomodel v1.5 user's manual. www.chronomodel.fr, 2016.

[21] A.G. Wintle and A.S. Murray. A review of quartz optically stimulated luminescence characteristics and their relevance in single-aliquot regeneration dating protocols. $R a$ diation Measurements, 41(4):369 - 391, 2006.

[22] A. Zink. A coarse Bayesian approach to evaluate luminescence ages. Geochronometria, 40(2):90-100, 2013.

\section{Appendix A. Construction of non-informative priors}

Appendix A.1. Jeffreys prior for $(\theta)$ with $Y \sim N(d(\theta) \cdot \alpha, d(\theta) \cdot \Sigma \cdot d(\theta))$ with $\alpha=\left[\alpha_{1}, \ldots, \alpha_{N}\right]^{T}$ and $\Sigma$ positive definite known

We denote $d(\boldsymbol{v})$ as the diagonal matrix whose diagonal entries are $v_{1}, \ldots, v_{N}$.

Using the fact that $d(\boldsymbol{\theta})$ is diagonal, a few algebra leads to rewrite the conditional density as:

$$
f_{Y}(\boldsymbol{y} \mid \boldsymbol{\theta}) \propto \frac{1}{\prod_{i} \theta_{i}} \exp \left(\left[d(\boldsymbol{\theta})^{-1} \Sigma^{-1} \boldsymbol{\alpha}, v_{\boldsymbol{\theta}}\right]^{T}\left[\boldsymbol{y}, v_{\boldsymbol{y}}\right]\right)
$$

where

$$
\begin{array}{r}
v_{\boldsymbol{\theta}}=-0.5\left[\Sigma_{1,1}^{-1} 1 / \theta_{1}^{2}, 2 \Sigma_{1,2}^{-1} 1 / \theta_{1} 1 / \theta_{2}, \ldots, 2 \Sigma_{1, N}^{-1} 1 / \theta_{1} 1 / \theta_{N}, \Sigma_{2,2}^{-1} 1 / \theta_{2}^{2}, \ldots, 2 \Sigma_{2, N}^{-1} 1 / \theta_{2} 1 / \theta_{N}\right. \\
\left.\ldots, \Sigma_{N-1, N-1}^{-1} 1 / \theta_{N-1}^{2}, \Sigma_{N-1, N}^{-1} 1 / \theta_{N-1} 1 / \theta_{N}, \Sigma_{N, N}^{-1} 1 / \theta_{N}^{2}\right]
\end{array}
$$

and

$$
v_{\boldsymbol{y}}=\left[y_{1}^{2}, y_{1} y_{2}, \ldots, y_{1} y_{N}, y_{2}^{2}, y_{2} y_{3}, \ldots, y_{2} y_{N}, \ldots y_{N-1}^{2}, y_{N-1} y_{N}, y_{N}^{2}\right]
$$

It thus constitutes an exponential family of dimension $\frac{N(N+3)}{2}$. 
By denoting $\boldsymbol{w}$ the vector composed of $\left[1 / \theta_{1}, \ldots, 1 / \theta_{N}\right]$, one can write the log-density as:

$$
l f(\boldsymbol{y}) \propto \sum_{i=1}^{N} \log \left(w_{i}\right)-\frac{1}{2}\left(\boldsymbol{w}^{T} d(\boldsymbol{y}) \Sigma^{-1} d(\boldsymbol{y}) \boldsymbol{w}\right)+\alpha^{T} \Sigma^{-1} d(\boldsymbol{y}) \boldsymbol{w},
$$

The Hessian matrix of the log-density $l f$ equals:

$$
\frac{\partial^{2} l f(\boldsymbol{y})}{\partial^{2} \boldsymbol{w}}=-d(\boldsymbol{y}) \Sigma^{-1} d(\boldsymbol{y})-d\left(\left[\frac{1}{w_{1}^{2}}, \ldots, \frac{1}{w_{N}^{2}}\right]\right) .
$$

After integration, we obtain

$$
E\left[\frac{\partial^{2} l f}{\partial^{2} \boldsymbol{w}} \mid \boldsymbol{w}\right]_{i, j}= \begin{cases}-\frac{1}{w_{i} w_{j}}\left[\Sigma_{i, j}^{-1}\left(\Sigma_{i, j}+\alpha_{i} \alpha_{j}\right)\right], & \text { if } i \neq j \\ -\frac{1}{w_{i}^{2}}\left[\Sigma_{i, i}^{-1}\left(\Sigma_{i, i}+\alpha_{i}^{2}\right)+1\right], & \text { if } i=j .\end{cases}
$$

Then,

$$
I(\boldsymbol{w})=-\operatorname{det}\left(E\left[\frac{\partial^{2} l f}{\partial \boldsymbol{w}^{2}} \mid \boldsymbol{w}\right]\right)=\operatorname{det}\left(d\left(\frac{1}{w_{i}}\right) \cdot C \cdot d\left(\frac{1}{w_{i}}\right)\right) \propto \prod_{i=1}^{N} \frac{1}{w_{i}^{2}},
$$

where $C$ is a $N \times N$ matrix with only positive entries and that does not depend from $\boldsymbol{w}$. Finally, one gets the density in $\theta$ by :

$$
p_{\Theta}(\boldsymbol{\theta}) \propto p_{W}\left(\left[1 / \theta_{1}, \ldots, 1 / \theta_{N}\right]\right) \prod_{i=1}^{N} \frac{1}{\theta_{i}^{2}} \propto \prod_{i=1}^{N}\left|\theta_{i}\right| \prod_{i=1}^{N} \frac{1}{\theta_{i}^{2}}=\prod_{i=1}^{N} \frac{1}{\left|\theta_{i}\right|} .
$$

Appendix A.2. Jeffreys prior for $\theta$ with $Y \sim N\left(\alpha \theta, \beta^{2} \theta^{2}+\sigma^{2}\right)$ with $\alpha$, $\beta$ and $\sigma$ known

The likelihood function can be write of the form

$$
f_{Y}(y \mid \theta) \propto \frac{e^{-\left(\alpha^{2} \theta^{2}\right) /\left(2\left(\beta^{2} \theta^{2}+\sigma^{2}\right)\right)}}{\sqrt{\beta^{2} \theta^{2}+\sigma^{2}}} e^{\left(y,-y^{2}\right)^{T}\left((\alpha \theta) /\left(\beta^{2} \theta^{2}+\sigma^{2}\right), 1 /\left(2\left(\beta^{2} \theta^{2}+\sigma^{2}\right)\right)\right.},
$$

so this model constitutes an exponential family of dimension 2. The log density is:

$$
l f(y)=-\frac{1}{2} \log \left(\beta^{2} \theta^{2}+\sigma^{2}\right)-\frac{1}{2} \log (2 \pi)-\frac{1}{2} \frac{(y-\alpha \theta)^{2}}{\beta^{2} \theta^{2}+\sigma^{2}},
$$

and

$$
\begin{aligned}
\frac{\partial^{2} l f}{\partial \theta^{2}} & =\frac{-\beta^{2}-\alpha^{2}}{\left(\sigma^{2}+\theta^{2} \beta^{2}\right)}+\frac{2 \theta^{2} \beta^{4}}{\left(\sigma^{2}+\theta^{2} \beta^{2}\right)^{2}}+(\theta \alpha-y) \frac{4 \theta \alpha \beta^{2}}{\left(\sigma^{2}+\theta^{2} \beta^{2}\right)^{2}} \\
& +(\theta \alpha-y)^{2}\left(\frac{\beta^{2}}{\left(\sigma^{2}+\theta^{2} \beta^{2}\right)^{2}}-\frac{4 \theta^{2} \beta^{4}}{\left(\sigma^{2}+\theta^{2} \beta^{2}\right)^{3}}\right)
\end{aligned}
$$


Finally,

$$
I(\theta)=-E\left[\frac{\partial^{2} l f}{\partial \theta^{2}} \mid \theta\right]=\frac{2 \beta^{4} \theta^{2}}{\left(\beta^{2} \theta^{2}+\sigma^{2}\right)^{2}}+\frac{\alpha^{2}}{\left(\beta^{2} \theta^{2}+\sigma^{2}\right)},
$$

and we obtain:

$$
p(\theta) \propto \sqrt{\frac{\theta^{2}\left(2 \beta^{4}+\alpha^{2} \beta^{2}\right)+\sigma^{2} \alpha^{2}}{\left(\theta^{2} \beta^{2}+\sigma^{2}\right)^{2}} .}
$$

When setting $\sigma=0$, one recovers $p(\theta) \propto \frac{1}{|\theta|}$.

\section{Appendix B. Building the covariances}

In this section, we show how to build the covariance matrix for a specific real example. Notice that the assumptions of independence involved in this particular example are not restrictive: all the quantities involved in the dosimetric data (that can be more numerous than in this particular example) can be composed of any numbers of independent and not independent error terms (as long as they remain gaussian).

As performed in the original publication of the studied data-set [19], we consider here

the dose-rate $\dot{d}_{i}$ as the sum of its $\beta, \gamma$ and cosmic components (the $\alpha$ component is supposed to be negligible):

$$
\dot{d}_{i}=\dot{d}_{\beta, i}+\dot{d}_{\gamma, i}+\dot{d}_{c o s, i}
$$

In practice, these three quantities are estimated through the combination of several physical quantities that are themselves not completely known. More specifically, for each sample $i$, one assesses values for the uranium, thorium and potassium contents $U_{i}, T h_{i}$ and $K_{i}$, the gamma radiation component $\gamma_{i}$, the cosmic radiation component $\cos _{i}$, the saturation uptake content $W_{i}$ and the fraction of average water content $F_{i}$. These six quantities are subject to the following errors:

- Measuring each of the $U_{i}, T h_{i}, K_{i}, \gamma$ and cosmic components of the dosimetry is subject to a zero-mean gaussian noise. Noises are independent between measurements. The corresponding standard deviations are known and called respectively, for each sample $i, \sigma_{U, i}, \sigma_{T h, i}, \sigma_{K, i}$ and $\sigma_{c o s, i}$. 
- Measuring the U, Th, K and $\gamma$ components of the dosimetry is subject to systematic percentage errors $\epsilon_{U, c}, \epsilon_{T h, c}, \epsilon_{K, c}$ and $\epsilon_{\gamma, c}$ of same variance $\sigma_{d, c}^{2}$ related to the calibration of the measurement device (typically $\sigma_{d, c}=0.1$ ) .

- The assessment of the product $W_{i} F_{i}$ is subject to an uncertainty modeled by a zeromean gaussian noise $\epsilon_{W F, i}$ of given standard deviation $\sigma_{W F, i}$ which is independent across samples.

Other sources of errors are considered as negligible.

The equations relating all these quantities to the $\beta, \gamma$ and cosmic dosimetries are the following [1]:

$$
\begin{aligned}
\forall i \in[|1, N|], \dot{d}_{\beta, i}= & \frac{1}{1+\psi_{\beta}\left(W_{i} F_{i}+\epsilon_{W F, i}\right)}\left(F_{\beta, U} S_{\beta, U}\left(U_{i}+\epsilon_{U, i}\right)\left(1+\epsilon_{U, c}\right)+\right. \\
& \left.F_{\beta, T h} S_{\beta, T h}\left(T h_{i}+\epsilon_{T h, i}\right)\left(1+\epsilon_{T h, c}\right)+F_{\beta, K} S_{\beta, K}\left(K_{i}+\epsilon_{K, i}\right)\left(1+\epsilon_{K, c}\right)\right)
\end{aligned}
$$

where $\psi_{\beta}$ is the absorption of the $\beta$ radiation by water, $F_{\beta, U}, F_{\beta, T h}$ and $F_{\beta, K}$ are the $\beta$ attenuation coefficients, $S_{\beta, U}, S_{\beta, T h}$ and $S_{\beta, K}$ are the specific beta dose rates,

$$
\dot{d}_{\gamma, i}=\left(\gamma_{i}+\epsilon_{\gamma, i}\right)\left(1+\epsilon_{\gamma, c}\right) \frac{1+\psi_{\gamma} W_{i}^{c} F_{i}^{c}}{1+\psi_{\gamma}\left(W_{i} F_{i}+\epsilon_{W F, i}\right)}
$$

where $\psi_{\gamma}$ is the absorption of the $\gamma$ radiation by water and $W_{i}^{c}$ and $F_{i}^{c}$ are respectively the saturation water uptake and the fractional average water at time of sampling,

$$
\dot{d}_{c o s, i}=\cos _{i}+\epsilon_{\cos , i}
$$

The noise terms $\epsilon_{U, c}, \epsilon_{T h, c}, \epsilon_{K, c}$ and $\epsilon_{\gamma, c}$ are common to the $N$ equations. Similarly to the common practice to compute $\dot{d}$ and its associated standard error, one then considers all error terms as independent and linearizes the previous error models to get the following age to characteristic equivalent-dose model:

$$
\forall i \in[1, N], D_{i}=A_{i}\left(\dot{d}_{i}+\epsilon_{\dot{d}, i}+\alpha_{i} \epsilon_{\dot{d}, c}\right)
$$


with

$$
\begin{aligned}
\dot{d}_{i}= & \frac{1}{1+\psi_{\beta} W_{i} F_{i}}\left(r_{U} U_{i}+r_{T h} T h_{i}+r_{K} K_{i}\right) \\
& \frac{1+\psi_{\gamma} W_{i}^{c} F_{i}^{c}}{1+\psi_{\gamma} W_{i} F_{i}} \gamma_{i}+\cos _{i}
\end{aligned}
$$

calling $r_{U}=F_{\beta, U} S_{\beta, U}, r_{T h}=F_{\beta, T h} S_{\beta, T h}$ and $r_{K}=F_{\beta, K} S_{\beta, K}$ and $\epsilon_{\dot{d}, i} \sim N\left(0, \sigma_{\dot{d}, i}^{2}\right)$ with:

$$
\begin{aligned}
\sigma_{\dot{d}, i}^{2}= & \frac{r_{U}^{2} \sigma_{i, U}^{2}+r_{T h}^{2} \sigma_{i, T h}^{2}+r_{K}^{2} \sigma_{i, K}^{2}}{\left(1+\psi_{\beta} W_{i} F\right)^{2}}+\frac{\left(1+\psi_{\gamma} W_{i}^{c} F_{i}^{c}\right)^{2} \sigma_{i, \gamma}^{2}}{\left(1+\psi_{\gamma} W_{i} F_{i}\right)^{2}}+ \\
& \sigma_{W_{i} F_{i}}^{2}\left(\frac{\psi_{\beta}}{\left(1+\psi_{\beta} W_{i} F_{i}\right)^{2}}\left(F_{\beta, U} S_{\beta, U} U_{i}+F_{\beta, T h} S_{\beta, T h} T h_{i}+F_{\beta, K} S_{\beta, K} K_{i}\right)+\right. \\
& \left.\frac{\gamma_{i} \psi_{\gamma}}{\left(1+\psi_{\gamma} W_{i} F_{i}\right)^{2}}\left(1+W_{i}^{c} F_{i}^{c}\right)\right)^{2} \\
& +\sigma_{c o s, i}^{2}
\end{aligned}
$$

and

$$
\alpha_{\dot{d}, i}^{2}=\frac{F_{\beta, U}^{2} S_{\beta, U}^{2} U_{i}^{2}+F_{\beta, T h}^{2} S_{\beta, T h}^{2} T h_{i}^{2}+F_{\beta, K}^{2} S_{\beta, K}^{2} K_{i}^{2}}{\left(1+\psi_{\beta} W_{i} F_{i}\right)^{2}}+\frac{\left(\left(1+\psi_{\gamma} W_{i}^{c} F_{i}^{c}\right) \gamma_{i}\right)^{2}}{\left(1+\psi_{\gamma} W_{i} F_{i}\right)^{2}}
$$

which completely defines the covariance matrix $\Sigma$.

\section{Acknowledgments}

The authors thank Guillaume Guérin, Chantal Tribolo and Norbert Mercier for introducing them the problem and providing and discussing the data of Section 4 . 